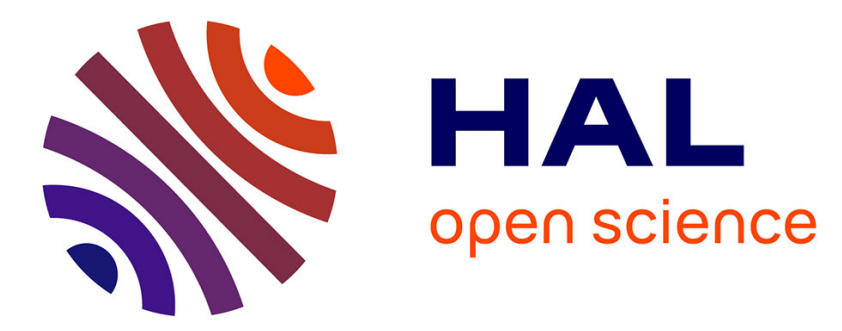

\title{
Bilan des enquêtes 2012, 2013 et 2014 de l'Observatoire national français des centres spécialisés de l'obésité (oNCSO)
}

Jean-Claude Desport, Anaïs Labrunie, Pierre-Marie Preux, E. Pencreach, O Ziegler

\section{To cite this version:}

Jean-Claude Desport, Anaïs Labrunie, Pierre-Marie Preux, E. Pencreach, O Ziegler. Bilan des enquêtes 2012, 2013 et 2014 de l'Observatoire national français des centres spécialisés de l'obésité (oNCSO). Cahiers de Nutrition et de Diététique, 2018, 53 (1), pp.34-42. 10.1016/j.cnd.2018.01.001 . hal-02341803

\section{HAL Id: hal-02341803 \\ https: / hal-unilim.archives-ouvertes.fr/hal-02341803}

Submitted on 3 Sep 2020

HAL is a multi-disciplinary open access archive for the deposit and dissemination of scientific research documents, whether they are published or not. The documents may come from teaching and research institutions in France or abroad, or from public or private research centers.
L'archive ouverte pluridisciplinaire HAL, est destinée au dépôt et à la diffusion de documents scientifiques de niveau recherche, publiés ou non, émanant des établissements d'enseignement et de recherche français ou étrangers, des laboratoires publics ou privés.

\section{(c)(1)}

Distributed under a Creative Commons Attribution| 4.0 International License 


\title{
Bilan des enquêtes 2012, 2013 et 2014 de l'Observatoire national français des centres spécialisés de l'obésité (oNCSO) 证, 败败
}

\section{Review of the 2012, 2013 and 2014 surveys of the French National Observatory of Specialized Centers for Obesity (oNCSO)}

\author{
Jean-Claude Desport ${ }^{a, *, b}$, Anaïs Labrunie ${ }^{b, c}$, \\ Pierre-Marie Preux ${ }^{\mathrm{b}, \mathrm{c}}$, Erell Pencreach ${ }^{\mathrm{d}}$, Olivier \\ Ziegler ${ }^{e}$, Direction Générale de l'Hospitalisation et \\ de l'Offre de Soins ${ }^{\mathrm{d}}$ Groupe de Concertation et de \\ Coordination (GCC) des $\mathrm{CSO}^{\mathrm{f}}$,
}

\author{
a Unité de nutrition et CSO du Limousin, CHU Dupuytren, avenue Martin-Luther-King, 87042 \\ Limoges cedex, France \\ b UMR Inserm 1094 NET, 2, rue du Dr-Marcland, 87025 Limoges cedex, France \\ c Unité de biostatistiques et de méthodologie de la recherche, $\mathrm{CHU}$, avenue \\ Martin-Luther-King, 87042 Limoges cedex, France \\ ${ }^{\mathrm{d}}$ DGOS, ministère des Solidarités et de la santé, 14, avenue Duquesne, 75350 Paris 07 SP, \\ France \\ e Unité multidisciplinaire de chirurgie de l'obésité (UMCO), hôpital Brabois-Adultes, CSO du \\ CHRU de Nancy, rue du Morvan, 54511 Vandoeuvre-les-Nancy, France \\ f GCC-CSO, Pr O. Ziegler, UMCO, hôpital Brabois-Adultes, CHRU de Nancy, rue du Morvan, \\ 54511 Vandoeuvre-les-Nancy, France
}

\begin{abstract}
Résumé
Objectif et méthode. - Le plan obésité a permis la création en France de 37 Centres Spécialisés de l'Obésité (CSO) en 2012 pour assurer une double mission, la prise en charge pluridisciplinaire de l'obésité sévère ou complexe et l'organisation des filières de soins dans les régions. Ce rapport fait le bilan des trois premières années de fonctionnement des CSO, à partir des données recueillies par l'observatoire national des CSO (oNCSO), mis en place par la Direction Générale de l'Hospitalisation et de l'Offre de Soins.
\end{abstract}

\footnotetext{
Ce travail a été présenté le 18 janvier 2017 à Toulouse lors des $33^{\text {e }}$ journées scientifiques de l'AFERO et le 4 octobre 2017 à Paris au ministère des Solidarités et de la santé lors du séminaire des CSO. L'article est publié conjointement dans les revues «Cahiers de Nutrition et Diététique », «Obésité » et « Nutrition Clinique et Métabolisme».

* Auteur correspondant.

Adresse e-mail : nutrition@unilim.fr (J.-C. Desport).
} 
Résultats. - Le bilan était globalement positif pour l'accès aux examens paracliniques, même si tous les CSO ne disposaient pas d'absorptiomètre biphotonique (DEXA) ou de calorimétrie. Les CSO développaient d'emblée des liens avec les 12 secteurs de prises en charge étudiés par l'oNCSO, avec quelques points faibles dont la psychiatrie. L'enquête ne permettait pas de faire le point sur les effectifs réels des CSO, au vu du nombre important de données aberrantes. Tous les CSO répondants déclaraient avoir des programmes d'éducation thérapeutique orientés vers les obèses pour les filières médicale, chirurgicale et pédiatrique. L'activité des CSO en médecine, chirurgie, gynécologie obstétrique et pédiatrie était hétérogène. En 2014, environ 25 à $30 \%$ de l'ensemble des interventions de chirurgie bariatrique étaient en France pratiqués dans les CSO. En moyenne les CSO recevaient environ 2500 patients adultes sévèrement obèses en consultation ou en hospitalisation de jour pour la filière médicale. Les résultats suggéraient une certaine fragilité des filières de gynécologie obstétrique et des filières pédiatriques.

Conclusion. - Cette enquête déclarative, malgré de nombreuses limites, montre cependant que les CSO ont pris d'emblée une place importante dans le système de soins franc, ais.

\begin{abstract}
Objective and method. - The French Obesity Plan enabled the creation of 37 Specialized Obesity Centers (CSOs) in 2012 to ensure a dual mission, the multidisciplinary management of severe or complex obesity and the organization of care channels in the regions. This report takes stock of the first three years of operation of the CSOs, based on the data collected by the National Observatory of CSOs (oNCSO), set up by the General Directorate of Hospitalization and Care. Results. - The overall results were positive for access to paraclinical examinations, although all CSOs did not have a biphotonic absorptiometer (DEXA) or calorimetry. The CSOs were initially developing links with the 12 sectors of care studied by the oNCSO, with some weaknesses including psychiatry. The survey did not make it possible to take stock of the real numbers of the actual workforce of the CSOs, given the large number of outliers. All responding CSOs reported having obese-oriented therapeutic education programs for the medical, surgical, and pediatric sectors. The activities of CSOs in medicine, surgery, gynecology and obstetrics were heterogeneous. In 2014, about $25-30 \%$ of all bariatric surgery procedures were performed in the CSOs in France. On average, CSOs received about 2500 severely obese adult patients in day care or in-patient care for the medical sector. The results suggested a certain fragility of the pathways of obstetric gynecology and the pediatric pathways.
\end{abstract}

Conclusion. - This declarative survey, despite many limitations, shows however that CSOs have taken an important place in the French care system.

\section{Introduction et objectif}

Avec $17,0 \%$ des adultes atteints selon la récente étude INCA 3 [1], l'obésité reste en France un problème de santé publique. L'instruction du 29 juillet 2011 relative à la mise en œuvre du Programme national nutrition santé (PNNS) 3 et du plan obésité [2] a permis d'identifier 37 Centres Spécialisés pour la prise en charge pluridisciplinaire de l'Obésité sévère (CSO) et pour l'organisation des filières de soins dans les régions. Afin de rendre l'offre de soins accessible et lisible pour les personnes obèses, ces CSO se sont vus confier deux missions principales s'inscrivant dans une logique de gradation des soins portée par les schémas organisationnels et plans régionaux de santé. La première mission est la prise en charge pluridisciplinaire de l'obésité sévère en recours territorial de $3^{\mathrm{e}}$ niveau. Ainsi, les CSO interviennent pour les situations les plus complexes et les soins spécifiques. Ces centres disposent de l'expertise (nutrition, endocrinologie-métabolisme, psychologie, diététique, etc.) et des équipements adaptés requis pour l'accueil des cas les plus difficiles en médecine, chirurgie et gynécologie-obstétrique. Ils collaborent étroitement avec des disciplines " clé » (pneumologie, centres du sommeil, cardiologie, hépato-gastroentérologie, etc.) et avec une équipe de chirurgie et d'anesthésie réanimation spécialisée en chirurgie bariatrique. La seconde mission est l'organisation de la filière de soins dans les territoires. Les CSO s'inscrivent dans une démarche d'animation, de coordination territoriale entre les acteurs, de formalisation de partenariats. Les centres ont pour tâche de contribuer, en lien avec les réseaux lorsqu'ils existent, à la coordination avec les acteurs du deuxième recours, notamment les 
Chirurgiens, la Médecine ambulatoire, les établissements autorisés en soins de suite et de réadaptation (SSR), les associations et les acteurs du médico-social. L'observatoire national des CSO (oNCSO) [3] est un système d'information qui permet la collecte d'informations thématiques directement auprès des établissements de santé, et met à leur disposition des restitutions dynamiques leur permettant de se comparer à des moyennes. L'observatoire est partagé entre les 37 centres spécialisés, les Agences Régionales de Santé (ARS) et l'administration centrale. L'objectif est de recueillir des indicateurs d'équipement, d'activité et de fonctionnement. L'intérêt de l'observatoire est d'animer un réseau de professionnels, de constituer un annuaire, de proposer un retour d'informations et de générer des statistiques comparatives.

L'objectif principal de l'étude était, à partir de trois collectes annuelles de données de l'oNCSO, de produire pour la Direction Générale de l'Offre de Soins (DGOS) du ministère français des Solidarités et de la santé une analyse quantitative descriptive et comparative du fonctionnement des CSO depuis leur mise en œuvre en 2012 jusqu'en 2014.

\section{Matériel et méthodes}

Une grille d'enquête électronique incluant des indicateurs d'activité, de fonctionnement et d'équipement était élaborée et transmise par la DGOS en 2013, 2014 et 2015 aux 37 CSO. Les données déclaratives étaient collectées par cette même Direction dans une base de données, puis transmises au Centre d'Epidémiologie, de Biostatistiques et de Méthodologie de la Recherche (CEBIMER) du CHU de Limoges pour analyse. Du point de vue statistique, le logiciel utilisé était SAS V9.3 (SAS Institute NC, Cary, États-Unis). Les résultats des analyses descriptives pour les variables continues étaient donnés en moyenne, écart-type, minimum et maximum, et pour les variables qualitatives en effectifs et pourcentages. Si l'analyse descriptive initiale montrait des résultats paraissant aberrants, les CSO en cause étaient contactés directement pour corrections. Dans le cas où les anomalies ne pouvaient être corrigées, les valeurs aberrantes étaient exclues. Les analyses comparatives évaluaient s'il y avait une différence de prise en charge des CSO au cours du temps (2012-2013-2014), selon un modèle GEE (Generalized Estimated Equation) avec une distribution binomiale pour les variables binaires à expliquer et un modèle multinomial pour les variables à expliquer qui avaient plus de deux modalités. La fonction de lien des modèles était la fonction Logit. Un effet aléatoire sur les CSO était également ajouté pour prendre en compte le fait que les données au cours du temps d'un même CSO n'étaient pas indépendantes. En cas d'effectif nul dans les catégories étudiées, la valeur de $p$ n'était pas estimable. Pour expliquer l'évolution des variables quantitatives, un modèle linéaire généralisé était utilisé sur données répétées. La distribution choisie était la distribution négative binomiale pour les variables de «comptage» (variable : «Nombre de ... par CSO ») et la fonction de lien était la fonction logarithme népérien. Le seuil de significativité pour l'ensemble des analyses était de 0,05.

\section{Résultats}

Un centre sur les 37 ne participait pas à l'étude en 2012. Tous les centres participaient en 2013 et 2014.
Tableau 1 Nombre de CSO ayant les équipements indispensables de 2012 à 2014.

\begin{tabular}{lcccc}
\hline $\begin{array}{l}\text { Équipements } \\
\text { indispensables } \\
\text { par CSO }\end{array}$ & $2012 n(\%)$ & $2013 n(\%)$ & $2014 n(\%)$ & $p$ \\
\hline $\begin{array}{l}0 \text { à } 4 \\
\text { équipements } \\
5 \text { à } 7\end{array}$ & $0(0 \%)$ & $0(0 \%)$ & $0(0 \%)$ & 0,84 \\
$\begin{array}{l}\text { équipements } \\
8 \text { à } 9\end{array}$ & $2(9 \%)$ & $2(8 \%)$ & $2(7 \%)$ & \\
$\begin{array}{l}\text { équipements } \\
10\end{array}$ & $7(32 \%)$ & $9(35 \%)$ & $10(36 \%)$ & \\
équipements & $13(59 \%)$ & $15(58 \%)$ & $16(57 \%)$ & \\
\hline
\end{tabular}

\section{1. Équipements et accès aux examens complémentaires}

\subsection{1. Équipements indispensables}

Les 10 équipements considérés comme indispensables par la DGOS étaient les suivants : système de pesée $>200 \mathrm{~kg}$, soulèves malade mobiles avec pesée, fauteuils d'accueil et de repos adaptés, brassards à tension adaptés, appareil polygraphique de dépistage, tables d'opération $>250 \mathrm{~kg}$, tables d'accouchement adaptée, tables d'examen de consultation adaptées, lits avec matelas anti-escarres, lits de réanimation avec pesée intégrée. Le nombre de CSO déclarant disposer de ces équipements de 2012 à 2014, selon le nombre d'équipements, est donné dans le Tableau 1. Aucun CSO ne disposait de moins de cinq équipements indispensables. Les lits de réanimation avec pesée intégrée n'étaient présents que dans $82 \%$ des cas en 2014. Il n'y avait pas d'évolution significative pour la période 2012-2014 en ce qui concerne le pourcentage de CSO équipés par les équipements indispensables.

L'évolution 2012-2014 en nombre d'équipements n'était positive et significative que pour les systèmes de pesée $>200 \mathrm{~kg}$, les soulèves malade mobiles avec pesée, les fauteuils d'accueil et de repos adaptés (Tableau 2 ).

\subsubsection{Autres équipements}

Des informations portant sur d'autres équipements adaptés aux personnes obèses étaient analysées dans les : la présence dans le CSO de matériel chirurgical, de brancards et fauteuils roulants, et la possibilité d'accès à un véhicule de transport bariatrique. En 2014, 97 \% des CSO avaient du matériel chirurgical adapté, $95 \%$ des brancards et fauteuils roulants adaptés, $49 \%$ avaient accès à un véhicule de transport bariatrique. L'évolution 2012-2014 n'était pas significative.

\subsubsection{Accès aux examens paracliniques}

Les accès aux examens paracliniques sont présentés par la Fig. 1. En 2014, 78 \% des CSO qui avaient répondu avaient accès à une IRM, $75 \%$ à une mesure de la composition corporelle par absorptiométrie biphotonique (DEXA), 62 \% à une mesure de la dépense énergétique de repos par calorimétrie. Il n'y avait pas de variation significative sur la période 2012-2014 mais on notait pour la DEXA une tendance à l'augmentation du nombre au cours des années de recueil (75\% en 2014 vs $59 \%$ en 2012, $p=0,08$ ). 
Tableau 2 Nombres des trois équipements pour lesquels il y avait une augmentation significative sur la période 20122014.

\begin{tabular}{lllll}
\hline $\begin{array}{l}\text { Équipements } \\
\text { indispensables }\end{array}$ & $\begin{array}{l}2012 \\
\text { moyenne } \pm \text { SD }(n) \\
{[\min ; \max ]}\end{array}$ & $\begin{array}{l}2013 \\
\text { moyenne } \pm \text { SD }(n) \\
{[\min ; \max ]}\end{array}$ & $\begin{array}{l}2014 \\
\text { moyenne } \pm \text { SD }(n) \\
{[\min ; \max ]}\end{array}$ \\
\hline $\begin{array}{l}\text { Systèmes de } \\
\text { pesée }>200 \mathrm{~kg}\end{array}$ & $\begin{array}{l}7,8 \pm 8,6(n=35) \\
{[0 ; 37]}\end{array}$ & $\begin{array}{l}9,7 \pm 14,2(n=37) \\
{[0 ; 70]}\end{array}$ & $\begin{array}{l}10,5 \pm 15,3 \\
(n=37)[0 ; 78]\end{array}$ \\
$\begin{array}{l}\text { Soulèves malades } \\
\text { mobiles avec }\end{array}$ & $9,6 \pm 14,9(n=34)$ & $\begin{array}{l}10,0 \pm 15,1 \\
(n=35)[0 ; 50]\end{array}$ & $\begin{array}{l}11,1 \pm 15,3 \\
(n=36)[0 ; 50]\end{array}$ \\
$\begin{array}{l}\text { pesée } \\
\begin{array}{l}\text { Fauteuils } \\
\text { d'accueil et de }\end{array}\end{array}$ & $\begin{array}{l}(0 ; 50] \\
\text { repos adaptés }\end{array}$ & $(n=30)[0 ; 122]$ & $\begin{array}{l}23,3 \pm 46,9 \\
(n=34)[0 ; 260]\end{array}$ & $\begin{array}{l}24,4 \pm 46,1 \\
(n=36)[0 ; 260]\end{array}$ \\
\hline
\end{tabular}

$n$ : nombre de CSO ayant répondu à la question.

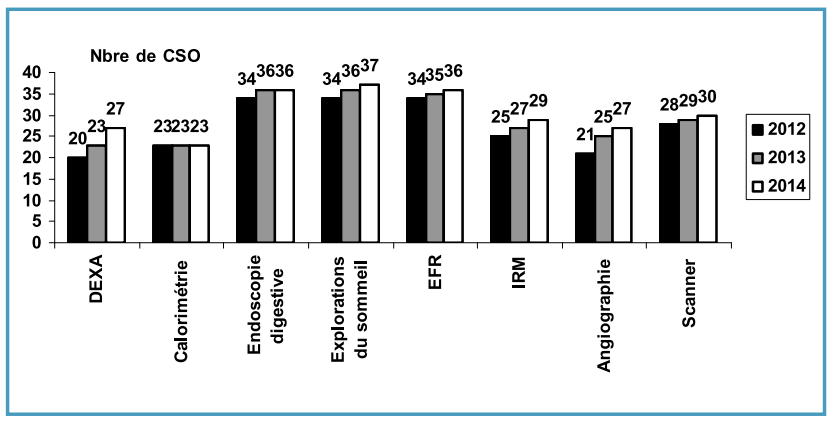

Fig. 1. Nombre de CSO déclarant avoir accès en 2012, 2013 et 2014 à divers examens paracliniques importants pour les personnes obèses. DEXA : absorptiométrie biphotonique ; EFR : explorations fonctionnelles respiratoires ; IRM : imagerie par résonnance magnétique nucléaire.

\subsection{Secteurs de prise en charge auxquels pouvaient faire appel les CSO}

La grille demandait des réponses vis-à-vis de 12 secteurs de prises en charge : gynécologie-obstétrique, psychiatrie, soins continus, cardiologie, réanimation, pneumologie, soins intensifs en cardiologie, hépatogastroentérologie, urgences médicales, urgences chirurgicales, consultation antidouleur, pédiatrie. Dès 2012, Plus de $80 \%$ des CSO bénéficiaient de 11 à 12 secteurs de prise en charge. Il n'y avait pas d'évolution significative pour la période 2012-2014. En 2014, c'était avec les secteurs de consultation antidouleur, de psychiatrie et les soins intensifs en cardiologie que les CSO avaient le moins de liens (liens présents dans respectivement $83 \%, 86 \%$ et $86 \%$ des cas).

\subsection{Effectifs des CSO}

La grille d'enquête devait recueillir en théorie le nombre et le temps de travail (en équivalents temps plein [ETP]) des divers professionnels médicaux, chirurgicaux, paramédicaux et techniques directement impliqués dans l'activité des CSO. Il est cependant certain que la compréhension de cette demande a été mauvaise, car de nombreux résultats d'effectifs maximaux (par exemple 114 infirmiers ou 34 médecins en 2014 pour un seul CSO) n'étaient pas réalistes. Ces résultats suggéraient que les établissements avaient donné des chiffres de personnels valables pour un établissement et non spécifiquement pour le CSO. Au vu du nombre

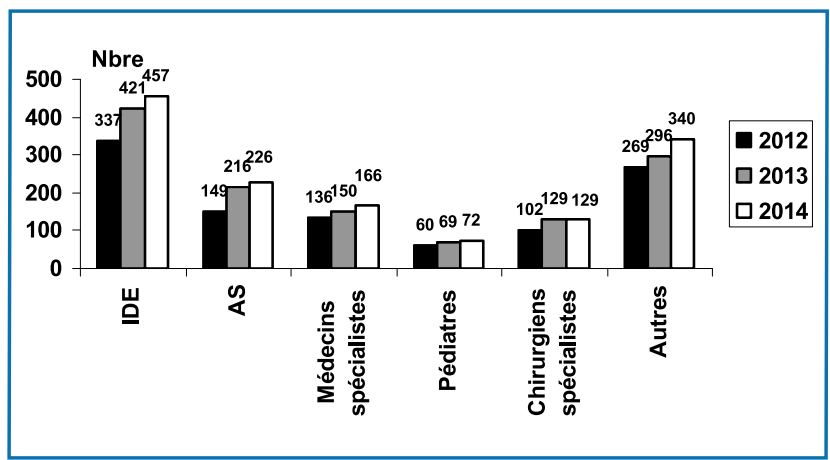

Fig. 2. Nombres cumulés des membres des diverses professions formés par les CSO en 2012, 2013 et 2014. IDE : infirmiers ; AS : aides-soignants.

important de données aberrantes, il était décidé de ne pas contacter les établissements concernés un à un, et les analyses n'étaient donc pas menées.

\subsection{Formation professionnelle des équipes par les CSO}

La grille demandait si les catégories suivantes de personnels avaient été formées : infirmiers (IDE), aides-soignants (AS), médecins spécialistes en nutrition, pédiatres, chirurgiens spécialistes, autres professionnels de santé. La Fig. 2 montre les nombres cumulés des membres des diverses professions formées en 2012, 2013 et 2014. Les variations n'étaient pas significatives.

En revanche, l'expression des résultats en nombre de personnels formés par CSO montrait une augmentation, mais uniquement pour la classe des médecins spécialistes en Nutrition $(3,7 \pm 3,8$ en $2012,4,0 \pm 4,0$ en $2013,4,4 \pm 4,3$ en $2014 ; p=0,006)$.

\subsection{Programmes d'éducation thérapeutique (ETP) du patient}

Tous les CSO ayant répondu à l'enquête en 2012, 2013 ou 2014 déclaraient disposer d'au moins un programme d'ETP validé par leur Agence régionale de santé, que ce soit dans le domaine médical, chirurgical ou pédiatrique. Cependant, concernant l'ETP en médecine, de 84 à $92 \%$ des CSO répondaient, selon les années, pour l'ETP en chirurgie, de 32 à 
$57 \%$ des CSO répondaient, et pour la Pédiatrie les réponses étaient données par 38 à $51 \%$ des CSO.

\subsection{Activités de consultations, hospitalisations, activité chirurgicale}

La grille d'enquête était segmentée en activités en médecine, chirurgie, gynécologie-obstétrique et pédiatrie. En 2014, étaient déclarés en médecine pour l'ensemble des CSO 48576 consultations pour obésité, 14356 séjours en hospitalisation complète, 27633 séjours en hôpital de jour, et 8546 dossiers avaient été discutés en réunion pluridisciplinaire. Le Tableau 3 montre les résultats de l'activité moyenne en médecine. Seul le nombre de séjours en hospitalisation de jour augmentait sur la période 2012-2014 $(p=0,03)$.

En 2014 étaient déclarées en chirurgie pour l'ensemble des CSO 41718 consultations pour obésité, 2170 poses d'anneaux, la réalisation de 4676 Sleeve Gastrectomies, de 4362 Bypass et de 2400 autres interventions. Une chirurgie de l'adolescent était déclarée dans 58 cas. Le Tableau 4 montre les résultats de l'activité moyenne en chirurgie. Le nombre total moyen d'interventions (hors chirurgie plastique) par CSO était de l'ordre de 260 en 2012, 350 en 2013 et 400 en 2014. Il existait une tendance à l'augmentation du nombre moyen de Sleeve gastrectomies pratiquées sur la période 2012-2014 ( $p=0,055)$.

En 2014, en Gynécologie-obstétrique le nombre cumulé de femmes suivies pour grossesse et ayant un $I M C>40 \mathrm{~kg} / \mathrm{m}^{2}$ pour l'ensemble des CSO était de 4803 . Le nombre déclaré d'enfants obèses suivis en pédiatrie était de 4800 (dont 58,6 \% avaient plus de 11 ans), le nombre d'hospitalisations complètes en Pédiatrie était de 1252, celui des hospitalisations de jour de 2300 et 511 enfants étaient adressés en SSR par les CSO. Le Tableau 5 montre les résultats de l'activité moyenne en gynécologie-obstétrique et en pédia- trie. Le nombre de femmes avec grossesse ayant bénéficié d'une CB avait tendance à augmenter sur la période 20122014 ( $p=0,052)$. En 2014, en moyenne, environ 200 enfants obèses par CSO étaient suivis.

\subsection{Fonctionnement des CSO}

Les modalités de fonctionnement des CSO déclarées en 2012, 2013 et 2014, en médecine, chirurgie et pédiatrie sont présentées dans le Tableau 6. En 2014, les lacunes en médecine portaient essentiellement sur les notices d'information destinées aux patients (absentes dans $27 \%$ des cas), les revues de morbi-mortalité (absentes dans 65 $\%$ des cas), le registre des patients suivis (absent dans $49 \%$ des cas). En chirurgie, les lacunes portaient sur les revues de morbi-mortalité (absentes dans $28 \%$ des cas). En pédiatrie la situation était moins favorable : absence de notice d'information-patients dans $38 \%$ des cas, de réunions d'information-patients dans $53 \%$ des cas, de RCP dans $32 \%$ des cas, de revues de morbi-mortalité dans $82 \%$ des cas et de registre patients dans $43 \%$ des cas. Il n'y avait aucune évolution significative au cours des années de recueil.

\subsection{Filières des CSO}

En 2014, l'ensemble des CSO déclarait collaborer avec 54 réseaux de santé spécialisés, 21 réseaux non spécialisés, et avoir organisé 177 rencontres avec des associations des patients. Par CSO, en 2014, ceci représentait $1,4 \pm 1,8$ $(n=37)$ réseaux spécialisés, $0,6 \pm 0,8 \quad(n=34)$ réseaux non spécialisés et 5,9 $\pm 6,3 \quad(n=30)$ rencontres. Il n'y avait aucune variation significative pour ces critères durant la période 2012-2014. Les CSO déclaraient par ailleurs des collaborations avec diverses organisations ou associations médicales ou chirurgicales, ainsi qu'avec des organisations

Tableau 3 Activité moyenne des CSO en médecine, en nombre de consultations, séjours, dossiers et comptes rendus de RCP, nombre de patients, en 2012, 2013 et 2014.

\begin{tabular}{|c|c|c|c|c|}
\hline Actes et patients & $\begin{array}{l}2012 \\
\text { Moyenne } \pm S D(n) \\
{[\min ; \max ]}\end{array}$ & $\begin{array}{l}2013 \\
\text { Moyenne } \pm S D(n) \\
{[\min ; \max ]}\end{array}$ & $\begin{array}{l}2014 \\
\text { Moyenne } \pm \text { SD }(n) \\
{[\min ; \max ]}\end{array}$ & $p$ \\
\hline Consultations & $\begin{array}{l}2222 \pm 2735 \\
(n=28) \\
{[45 ; 11924]}\end{array}$ & $\begin{array}{l}1870 \pm 3108 \\
(n=30) \\
{[169 ; 15733]}\end{array}$ & $\begin{array}{l}1619 \pm 2217 \\
(n=30) \\
{[189 ; 8876]}\end{array}$ & 0,15 \\
\hline Séjours en hospitalisation complète & $\begin{array}{l}524 \pm 794 \\
(n=30) \\
{[0 ; 3367]}\end{array}$ & $\begin{array}{l}379 \pm 433 \\
(n=29) \\
{[0 ; 1465]}\end{array}$ & $\begin{array}{l}435 \pm 483 \\
(n=33) \\
{[0 ; 1508]}\end{array}$ & 0,23 \\
\hline Séjours en hospitalisation de jour & $\begin{array}{l}631 \pm 794 \\
(n=32) \\
{[0 ; 3251]}\end{array}$ & $\begin{array}{l}731 \pm 731 \\
(n=29) \\
{[10 ; 2533]}\end{array}$ & $\begin{array}{l}813 \pm 804 \\
(n=34) \\
{[44 ; 2937]}\end{array}$ & 0,03 \\
\hline Dossiers discutés en RCP & $\begin{array}{l}282 \pm 250 \\
(n=33) \\
{[0 ; 797]}\end{array}$ & $\begin{array}{l}305 \pm 229 \\
(n=31) \\
{[0 ; 814]}\end{array}$ & $\begin{array}{l}267 \pm 216 \\
(n=32) \\
{[27 ; 814]}\end{array}$ & 0,23 \\
\hline Comptes rendus de RCP & $\begin{array}{l}263 \pm 263 \\
(n=32) \\
{[0 ; 770]}\end{array}$ & $\begin{array}{l}261 \pm 240 \\
(n=32) \\
{[0 ; 814]}\end{array}$ & $\begin{array}{l}248 \pm 218 \\
(n=31) \\
{[3 ; 814]}\end{array}$ & 0,96 \\
\hline Patients adressés en SSR & $\begin{array}{l}58 \pm 82 \\
(n=20) \\
{[0 ; 276]}\end{array}$ & $\begin{array}{l}72 \pm 99 \\
(n=25) \\
{[0 ; 363]}\end{array}$ & $\begin{array}{l}66 \pm 89 \\
(n=25) \\
{[1 ; 360]}\end{array}$ & 0,96 \\
\hline
\end{tabular}

$n$ : nombre de CSO ayant répondu à la question ; RCP : réunion de concertation pluridisciplinaire ; SSR : service de soins de suite et de réadaptation. 
Tableau 4 Activité moyenne des CSO en chirurgie, en nombre d'actes, en 2012, 2013 et 2014.

\begin{tabular}{|c|c|c|c|c|}
\hline Actes & $\begin{array}{l}2012 \\
\text { Moyenne } \pm \text { SD }(n) \\
{[\min ; \max ]}\end{array}$ & $\begin{array}{l}2013 \\
\text { Moyenne } \pm \text { SD }(n) \\
{[\min ; \max ]}\end{array}$ & $\begin{array}{l}2014 \\
\text { Moyenne } \pm \text { SD }(n) \\
{[\min ; \max ]}\end{array}$ & $p$ \\
\hline Consultations & $\begin{array}{l}1440 \pm 1131 \\
(n=26) \\
{[170 ; 4308]}\end{array}$ & $\begin{array}{l}1405 \pm 1828 \\
(n=29) \\
{[50 ; 9325]}\end{array}$ & $\begin{array}{l}1490 \pm 1818 \\
(n=28) \\
{[80 ; 8519]}\end{array}$ & 0,64 \\
\hline Anneaux gastriques & $\begin{array}{l}26 \pm 50 \\
(n=31)[0 ; 162]\end{array}$ & $\begin{array}{l}66 \pm 207 \\
(n=33)[0 ; 1173]\end{array}$ & $\begin{array}{l}64 \pm 255 \\
(n=34)[0 ; 1484]\end{array}$ & 0,14 \\
\hline Sleeve gastrectomies & $\begin{array}{l}110 \pm 142 \\
(n=32)[2 ; 593]\end{array}$ & $\begin{array}{l}118 \pm 164 \\
(n=33)[6 ; 645]\end{array}$ & $\begin{array}{l}134 \pm 188 \\
(n=35)[6 ; 871]\end{array}$ & 0,055 \\
\hline Bypass & $\begin{array}{l}97 \pm 117 \\
(n=32)[0 ; 434]\end{array}$ & $\begin{array}{l}128 \pm 124 \\
(n=32)[0 ; 443]\end{array}$ & $\begin{array}{l}125 \pm 132 \\
(n=35)[0 ; 434]\end{array}$ & 0,07 \\
\hline Autres interventions & $\begin{array}{l}26 \pm 29 \\
(n=30)[0 ; 113]\end{array}$ & $\begin{array}{l}39 \pm 94 \\
(n=32)[0 ; 523]\end{array}$ & $\begin{array}{l}73 \pm 295 \\
(n=33)[0 ; 1713]\end{array}$ & 0,56 \\
\hline Interventions chez des adolescents & $\begin{array}{l}2,2 \pm 8,4 \\
(n=24)[0 ; 40]\end{array}$ & $\begin{array}{l}1,5 \pm 3,8 \\
(n=29)[0 ; 15]\end{array}$ & $\begin{array}{l}1,7 \pm 4,3 \\
(n=33)[0 ; 20]\end{array}$ & 0,93 \\
\hline Interventions en chirurgie plastique & $\begin{array}{l}62 \pm 89 \\
(n=22)[0 ; 423]\end{array}$ & $\begin{array}{l}48 \pm 53 \\
(n=20)[0 ; 159]\end{array}$ & $\begin{array}{l}57 \pm 53 \\
(n=23)[0 ; 181]\end{array}$ & 0,89 \\
\hline
\end{tabular}

$n:$ nombre de CSO ayant répondu à la question.

Tableau 5 Activité moyenne des CSO en gynécologie-obstétrique et en pédiatrie, en nombre de patients, en 2012,2013 et 2014.

\begin{tabular}{|c|c|c|c|c|}
\hline Types de patients & $\begin{array}{l}2012 \\
\text { Moyenne } \pm \text { SD }(n) \\
{[\min ; \max ]}\end{array}$ & $\begin{array}{l}2013 \\
\text { Moyenne } \pm \text { SD }(n) \\
{[\min ; \max ]}\end{array}$ & $\begin{array}{l}2014 \\
\text { Moyenne } \pm \text { SD }(n) \\
{[\min ; \max ]}\end{array}$ & $p$ \\
\hline $\begin{array}{l}\text { Femmes enceintes suivies } \\
\text { avec } \mathrm{IMC}>40 \mathrm{~kg} / \mathrm{m}^{2} \text { sans } \\
\text { chirurgie bariatrique }\end{array}$ & $\begin{array}{l}155 \pm 237 \\
(n=20)[0 ; 975]\end{array}$ & $\begin{array}{l}122 \pm 158 \\
(n=22)[0 ; 461]\end{array}$ & $\begin{array}{l}174 \pm 198 \\
(n=25)[0 ; 566]\end{array}$ & 0,11 \\
\hline $\begin{array}{l}\text { Femmes enceintes suivies } \\
\text { ayant eu une chirurgie } \\
\text { bariatrique }\end{array}$ & $\begin{array}{l}13 \pm 23 \\
(n=18)[0 ; 100]\end{array}$ & $\begin{array}{l}22 \pm 31 \\
(n=17)[0 ; 120]\end{array}$ & $\begin{array}{l}22 \pm 30 \\
(n=20)[0 ; 120]\end{array}$ & 0,052 \\
\hline $\begin{array}{l}\text { Enfants obèses suivis } \\
\text { d'âge }<11 \text { ans }\end{array}$ & $\begin{array}{l}75 \pm 90 \\
(n=16)[0 ; 300]\end{array}$ & $\begin{array}{l}78 \pm 82 \\
(n=20)[0 ; 315]\end{array}$ & $\begin{array}{l}79 \pm 78 \\
(n=25)[0 ; 315]\end{array}$ & 0,50 \\
\hline $\begin{array}{l}\text { Enfants obèses suivis } \\
\text { d'âge }>11 \text { ans }\end{array}$ & $\begin{array}{l}105 \pm 184 \\
(n=17)[0 ; 780]\end{array}$ & $\begin{array}{l}187 \pm 286 \\
(n=20)[0 ; 986]\end{array}$ & $\begin{array}{l}113 \pm 127 \\
(n=25)[0 ; 488]\end{array}$ & 0,06 \\
\hline
\end{tabular}

$n$ : nombre de CSO ayant répondu à la question ; IMC : indice de masse corporelle.

ou associations d'activité physique adaptée (APA) ou de diététiciens. Les résultats sont donnés dans le Tableau 7.

\section{Discussion}

Avec ces enquêtes, nous disposons pour la première fois d'éléments transversaux et longitudinaux (résultats en 2012, 2013 et 2014) portant sur l'activité des 37 CSO français.

Les résultats montrent l'importance des CSO dans les parcours de soins en France pour les patients obèses, que ce soit en nombre de consultations, de journées d'hospitalisation ou d'interventions chirurgicale. Ils montrent également que les équipements requis pour la prise en charge de l'obésité sont le plus souvent présents dans les CSO, que ceux-ci ont bien intégré l'intérêt de l'activité d'ETP, fondamentale pour ces patients [4], et qu'ils ont également de nombreux liens avec des associations extrahospitalières.
Cette étude a des limites. C'est ainsi que la première année a débuté officiellement pour certains CSO en février 2012, donc n'a inclus que 11 mois, et pour d'autres CSO l'installation n'a eu lieu que dans le courant de cette même année, donc les résultats ont pu être minorés par rapport aux années suivantes. De plus, certaines données ont pu être d'obtention difficile du point de vue administratif, ce qui fait que tous les CSO n'ont pas répondu à toutes les questions. De plus, certaines questions avaient une formulation qui pouvait prêter à confusion. Ces insuffisances pourraient être en partie corrigées à l'avenir. Par ailleurs, les données transmises étaient bien entendu déclaratives. Elles n'ont fait l'objet de vérifications et parfois de corrections que de manière ponctuelle, lorsque des résultats semblaient très anormaux. Dans certains cas, les données n'ont pas pu être analysées.

$\mathrm{Au}$ sujet des équipements, l'équipement technique adapté aux personnes obèses dans les CSO paraissait globalement satisfaisant, avec une exception pour les lits de réanimation avec pesée intégrée. Cependant, il est possible 
Tableau 6 Modalités de fonctionnement des CSO déclarées en 2012, 2013 et 2014, en médecine, chirurgie et pédiatrie, en nombre de CSO ayant répondu positivement et pourcentage par rapport au nombre total de CSO répondeurs.

\begin{tabular}{|c|c|c|c|c|}
\hline Modalité & $\begin{array}{l}2012 \\
n(\%)\end{array}$ & $\begin{array}{l}2013 \\
n(\%)\end{array}$ & $\begin{array}{l}2014 \\
n(\%)\end{array}$ & $p$ \\
\hline \multicolumn{5}{|l|}{ Médecine } \\
\hline Parcours personnalisés de soins & $33 / 36(92 \%)$ & $35 / 37$ (95 \%) & $36 / 37$ (97 \%) & 0,37 \\
\hline Réunions d’information & $26 / 35$ (74 \%) & $27 / 37$ (73 \%) & 29/37 (78 \%) & 0,24 \\
\hline Notices d'information & $23 / 35(66 \%)$ & $24 / 37(65 \%)$ & $27 / 37$ (73\%) & 0,22 \\
\hline $\mathrm{RCP}$ & $34 / 35(97 \%)$ & $36 / 36(100 \%)$ & $36 / 36(100 \%)$ & NE \\
\hline Comptes rendus de RCP transmis au médecin traitant & $30 / 35(86 \%)$ & $32 / 36(89 \%)$ & $34 / 36(94 \%)$ & 0,22 \\
\hline Revues de morbi-mortalité & $10 / 35(29 \%)$ & $13 / 37(35 \%)$ & $13 / 37$ (35 \%) & 0,25 \\
\hline Dossier médical informatisé & 25/35 (71 \%) & $28 / 37(76 \%)$ & 29/37 (78 \%) & 0,23 \\
\hline Dossier médical accessible en urgence & $32 / 34(94 \%)$ & $37 / 37(100 \%)$ & $37 / 37(100 \%)$ & NE \\
\hline Registre des patients suivis & $17 / 35(49 \%)$ & $18 / 37(49 \%)$ & $19 / 37(51 \%)$ & 0,53 \\
\hline \multicolumn{5}{|l|}{ Chirurgie } \\
\hline Parcours personnalisés de soins & $33 / 36(92 \%)$ & $35 / 37$ (95 \%) & $36 / 37$ (97 \%) & 0,37 \\
\hline Réunions d'information & $26 / 34(76 \%)$ & $27 / 36(75 \%)$ & $28 / 36(78 \%)$ & 0,57 \\
\hline Notices d'information & $33 / 35(94 \%)$ & $33 / 37(89 \%)$ & $34 / 37(92 \%)$ & 0,38 \\
\hline $\mathrm{RCP}$ & $36 / 36(100 \%)$ & $37 / 37(100 \%)$ & $37 / 37(100 \%)$ & NE \\
\hline Comptes rendus de RCP transmis au médecin traitant & $32 / 36(89 \%)$ & $34 / 37(92 \%)$ & $36 / 37(97 \%)$ & 0,22 \\
\hline Revues de morbi-mortalité & $22 / 29(76 \%)$ & $25 / 32(78 \%)$ & $23 / 32(72 \%)$ & 0,28 \\
\hline Dossier médical informatisé & 24/34 (71 \%) & $27 / 36(75 \%)$ & $28 / 36(78 \%)$ & 0,23 \\
\hline Dossier médical accessible en urgence & $32 / 33(97 \%)$ & $35 / 36(97 \%)$ & $35 / 36(97 \%)$ & 0,61 \\
\hline Registre des patients suivis & $27 / 32(84 \%)$ & $28 / 35(80 \%)$ & $30 / 35(86 \%)$ & 0,37 \\
\hline \multicolumn{5}{|l|}{ Pédiatrie } \\
\hline Parcours personnalisés de soins & $20 / 26(77 \%)$ & $25 / 30(83 \%)$ & $26 / 30(87 \%)$ & 0,24 \\
\hline Réunions d'information & $12 / 22(55 \%)$ & $14 / 28(50 \%)$ & $14 / 30(47 \%)$ & 0,43 \\
\hline Notices d'information & $11 / 22(50 \%)$ & $14 / 27$ (52 \%) & $18 / 29(62 \%)$ & 0,23 \\
\hline $\mathrm{RCP}$ & $17 / 26(65 \%)$ & $21 / 31(68 \%)$ & $21 / 31(68 \%)$ & 0,99 \\
\hline Comptes rendus de RCP transmis au médecin traitant & $12 / 20(60 \%)$ & $16 / 28(57 \%)$ & $16 / 28(57 \%)$ & 0,63 \\
\hline Revues de morbi-mortalité & $1 / 20(5 \%)$ & $4 / 26(15 \%)$ & $5 / 28(18 \%)$ & 0,11 \\
\hline Dossier médical informatisé & $14 / 25(56 \%)$ & $20 / 29(69 \%)$ & $22 / 30(73 \%)$ & 0,13 \\
\hline Dossier médical accessible en urgence & $23 / 24(96 \%)$ & $27 / 28(96 \%)$ & $27 / 29(93 \%)$ & 0,43 \\
\hline Registre des patients suivis & $12 / 22(55 \%)$ & $17 / 27(63 \%)$ & $16 / 28(57 \%)$ & 0,32 \\
\hline
\end{tabular}

RCP : réunion de concertation pluridisciplinaire ; NE : non évaluable.

Tableau 7 Type d'association ou organisation avec lesquelles les CSO déclaraient être en lien en 2012, 2013 et 2014, en nombre de CSO ayant répondu positivement et pourcentage par rapport au nombre total de CSO répondeurs.

\begin{tabular}{lcccr}
\hline Type d'association ou organisation & \multicolumn{1}{c}{$\begin{array}{l}2012 \\
n(\%)\end{array}$} & $\begin{array}{l}2013 \\
n(\%)\end{array}$ & $\begin{array}{l}2014 \\
n(\%)\end{array}$ & $p$ \\
\hline Spécialisée médicale & $18 / 27(67 \%)$ & $22 / 33(67 \%)$ & $21 / 33(63 \%)$ & 0,60 \\
Spécialisée chirurgicale & $9 / 25(36 \%)$ & $14 / 32(44 \%)$ & $15 / 32(47 \%)$ & 0,33 \\
Activité physique adaptée & $22 / 30(73 \%)$ & $28 / 35(80 \%)$ & $32 / 36(89 \%)$ & 0,09 \\
Diététiciens & $17 / 31(55 \%)$ & $21 / 34(62 \%)$ & $25 / 35(71 \%)$ & 0,10 \\
\hline
\end{tabular}

que certains CSO n'aient pas eu de service de réanimation dans la structure même, et donc n'aient pas pu répondre positivement à la question. Une progression significative était notée de 2012 à 2014 en ce qui concerne l'équipement en systèmes de pesée à plus de $200 \mathrm{~kg}$, en lèves malade mobiles avec pesée et en fauteuils d'accueil et de repos adaptés aux obèses. Néanmoins, certains CSO déclaraient ne pas être équipés du tout pour certains appareillages, même pour des appareillages de base. Ces derniers résultats mériteraient des vérifications pour la situation actuelle. Un équipement pour le transport des grands obèses (véhicules de transport bariatrique) n'était déclaré, même en 2014, que dans environ $50 \%$ des cas, suggérant un déficit important sur ce point. Il est cependant probable que la situation se soit améliorée à la suite de la directive souhaitant promouvoir les équipements pour transports bariatriques dans les régions [3]. Ceci serait également à vérifier.

Concernant les équipements paracliniques, relativement peu de CSO étaient équipés d'absorptiomètres (DEXA), mais on notait une tendance à l'augmentation du nombre au cours des années de recueil ( $59 \%$ des CSO en 2012 vs $75 \%$ en 2014, $p=0,08)$. Relativement peu de CSO étaient équipés de calorimètres (62\% en 2014), bien qu'il s'agisse d'un appareillage fondamental pour les bilans de certaines personnes obèses [5]. Enfin, il n'était pas précisé dans les réponses si les scanners et les IRM étaient adaptés aux grands obèses ou non, ce qui serait à vérifier, ainsi que l'accès possible ou non à une impédancemétrie. 
Au sujet des secteurs de prises en charge dont pouvaient bénéficier les patients suivis dans les CSO, l'absence dans le questionnaire de repérage des services d'endocrinologie et/ou de nutrition et/ou de diététique ni des secteurs de prise en charge de l'activité physique adaptée (APA), qui constituent pourtant la base des CSO, pourrait aisément être corrigé dans une enquête future. Certains CSO déclaraient ne pas pouvoir faire appel à un secteur de psychiatrie (encore dans $14 \%$ des cas en 2014), ce qui paraît constituer un manque, ou à un secteur de prise en charge de la douleur (dans $17 \%$ des cas), et là aussi il y avait probablement une lacune. L'absence de lien entre un CSO et un secteur de psychiatrie est surprenante, compte tenu des recommandations professionnelles qui intègrent la nécessité d'une prise en charge psychologique et psychiatrique dans le parcours de soins des patients [6]. Ce problème a pourtant déjà été signalé spécifiquement pour la chirurgie bariatrique dans les CSO [7]. Il est cependant possible qu'y il ait eu une ambiguité pour les CSO qui ont pu confondre dans leurs réponses prise en charge psychiatrique et prise en charge psychologique.

$\mathrm{Au}$ sujet des programmes d'éducation thérapeutique, tous les CSO répondants déclaraient avoir des programmes orientés vers les obèses pour les filières médicale, chirurgicale et pédiatrique et validés par les ARS, ce qui est un facteur très positif, soulignant une fois de plus l'intérêt de l'ETP pour le traitement de l'obésité [4]. Néanmoins, le nombre de CSO n'ayant pas répondu est élevé, et cette activité pourrait être réévaluée.

Au sujet de l'activité des CSO en médecine, chirurgie, gynécologie-obstétrique et pédiatrie, on constatait globalement en médecine une grande disparité des résultats selon les CSO. Ainsi en 2014 les extrêmes pour le nombre de consultations en médecine étaient de 189 et 8876 , le nombre de dossiers discutés en RCP allait de 27 à 814 , le nombre de patients adressés en SSR allait de 1 à 360 , et un CSO au moins déclarait n'avoir fait aucune hospitalisation complète pour obésité. Ceci suggère des déclarations erronées, mais surtout que les différents CSO ont des niveaux d'activités très variables. Seul le nombre de séjours en hospitalisation de jour pour obésité par CSO était plus élevé en 2014 par rapport à 2012 et à 2013, ce qui souligne l'importance de cette modalité de prise en charge, déjà bien identifiée dans les CSO [8]. En chirurgie, les résultats étaient là encore très disparates, signant des activités très différentes d'un CSO à l'autre. Toutes interventions confondues, environ 13600 interventions étaient pratiquées dans les CSO pour obésité, soit 25 à $30 \%$ de l'ensemble des interventions du même type en France [9], alors que les 37 CSO représentaient seulement environ $8,5 \%$ des lieux de chirurgie bariatrique dans notre pays en 2013 [9]. Ceci témoigne du rôle majeur des CSO dans l'offre de chirurgie bariatrique. On observait une évolution surprenante du type de chirurgie, avec un niveau stable des poses d'anneau entre 2012 et 2014 et une tendance à la hausse pour les Sleeve gastrectomies uniquement. Seul ce dernier point semble conforme à l'évolution générale française [9]. L'activité globale pour les femmes enceintes obèses et en pédiatrie semblait très disparate, avec par exemple un CSO sans aucune activité, quel que soit le critère. Par ailleurs, le nombre de CSO n'ayant pas répondu était important. Le tout suggérait que les codages pouvaient être défaillants et plus généralement que les filières pour ces populations étaient fragiles [10].

$\mathrm{Au}$ sujet de la structuration du fonctionnement des CSO en médecine, chirurgie et pédiatrie, les lacunes en médecine semblaient être l'insuffisance de revues de morbimortalités (absentes en 2014 dans 65 \% des CSO), l'absence d'un registre des patients suivis (dans $49 \%$ des CSO), et à un moindre degré l'absence de notices d'information destinées aux patients (dans $27 \%$ des CSO). En chirurgie, la structuration était plus solide, et seules les revues de morbi-mortalité étaient absentes dans $28 \%$ des CSO. C'est en pédiatrie que les problèmes étaient les plus nombreux : déficit en terme de réunions d'information patients (absentes dans $53 \%$ des CSO), de notices d'information (absentes dans $38 \%$ des CSO), de RCP (absentes dans 32 $\%$ des (SO), de transmissions des RCP aux médecins traitants (absentes dans $43 \%$ des CSO) et de registre des patients (absent dans $43 \%$ des CSO). La fragilité de la filière pédiatrique était une fois de plus soulignée [10]. Cette constatation pourrait être expliquée par une moindre valorisation financière de l'activité médicale par rapport à l'activité chirurgicale (essentiellement présente chez l'adulte).

Au sujet des collaborations des CSO avec les réseaux de soins et les associations de patients, le nombre de rencontres avec les associations de patients était très favorable (six rencontres par CSO en moyenne en 2014). Le nombre de CSO ayant des collaborations avec les associations d'APA et les associations de diététiciens tendait à augmenter de 2012 à 2014, à la limite de la significativité ( $p=0,08$ et 0,09 respectivement), ce qui parait positif.

\section{Conclusion}

Cette enquête permet d'avoir une vision globale sur les équipements et les activités des CSO en France entre 2012 et 2014. Elle montre que cette structuration de l'offre de soins autour des CSO a eu rapidement un impact sur le parcours de soins des patients sévèrement obèses. Certains points pourraient être éclaircis, en particulier en ce qui concerne le nombre et le temps de travail des professionnels spécifiquement affectés aux CSO. Des compléments d'information seraient intéressants portant sur les activités de recherche clinique ou fondamentale des CSO ou des Centres Intégrés de l'Obésité, qui peuvent être adossés aux CSO. De manière indirecte, les résultats suggèrent une certaine fragilité des filières de gynécologie-obstétrique pour les personnes obèses ainsi que des filières pédiatriques, qui mériteraient probablement d'être renforcées.

\section{Déclaration de liens d'intérêts}

Les auteurs déclarent ne pas avoir de liens d'intérêts.

\section{Références}

[1] Anon. Étude individuelle nationale des consommations alimentaires 3 (INCA 3). Avis de l'Anses rapport d'expertise collective; 2017 [https://www.anses.fr/ $\mathrm{fr} /$ system/files/NUT2014SA0234Ra.pdf].

[2] Ministère des Solidarités et de la santé. Instruction DGS/EA3/DGOS n²011-380 du 29 juillet 2011 relative à la mise en œuvre du programme national nutrition santé (PNNS 3) et du plan obésité (PO) par les agences régionales de santé (ARS); 2011 [http://solidarites-sante.gouv. $\mathrm{fr} /$ fichiers/bo/2011/11-12/ste_20110012_0100_0084.pdf].

[3] Ministère des Solidarités et de la santé. Instruction $\mathrm{N}^{\circ}$ DGOS/R4/2013/222 du 3 juin 2013 relative à la mise en œuvre des actions 4 et 10 du plan obésité; 2013 [http://circulaires.legifrance.gouv.fr/pdf/2013/06/ 
cir_37065.pdf; AFERO. Observatoire oNCSO. http://www. afero.fr/FR/centres_specialistes_de_l_obesite/lobservatoire_ oncso.asp].

[4] Ziegler O, Bertin E, Jouret B, Calvar R, Sanguignol F, Avignon $\mathrm{A}$, et al. Éducation thérapeutique et parcours de soins de la personne obèse. Référentiel et organisation. Rapport à la Direction générale de la santé et à la Direction générale de l'offre de soins. Obésité 2014;9:302-28.

[5] Pivois L, Frutel A, Fayemendy P, Nassouri S, Lopez S, Galinat $S$, et al. Comparaison de la mesure de la dépense énergétique de repos par calorimétrie indirecte à plusieurs formules de la littérature ainsi qu'aux niveaux énergétiques de régime proposés par le bilan diététique dans une cohorte de patients obèses. Cah Nutr Diet 2012;47:139-46.

[6] HAS. Surpoids et obésité de l'adulte : prise en charge médicale de premier recours; 2011 [https://www.hassante. fr/portail/upload/docs/application/pdf/2011-10/ reco2clics_obesite_adulte_premiers_recours.pdf].
[7] Lamore K, Kaci SS, Czernichow S, Bretault M, Bouillot JL, Naudé AJ, et al. Mental health support provided throughout the bariatric surgery clinical pathway in French specialized Care Centers for Obesity. Obes Surg 2017;27:802-10.

[8] Delcourt A, Andrieux S, Gueorguieva I, Lepage M, VérierMine $\mathrm{O}$, Romon $\mathrm{M}$, et al. Prise en charge de l'obésité sévère de l'adulte dans les Centres spécialisés de l'obésité. Place et fonction de l'hospitalisation de courte durée. Propositions du Groupe de concertation et de coordination national des «Centres spécialisés de l'obésité ». Obésité 2015;10: 293-305.

[9] Schaaf C, Lannelli A, Gugenheim J. État actuel de la chirurgie bariatrique en France. e-mémoires de l'Académie nationale de chirurgie, 14; 2015. p. 104-7.

[10] HAS. Surpoids et obésité de l'enfant et de l'adolescent; 2011 [https://www.has-sante.fr/portail/upload/docs/application/ pdf/2011-10/reco2clics_obesite_enfant_adolescent.pdf]. 